\title{
On some properties of linear and nonlinear waves in pair-ion plasmas
}

\author{
H. Saleem ${ }^{\text {a,b,c, }, *}$ J. Vranjes ${ }^{\text {a }}$, S. Poedts ${ }^{\text {a }}$ \\ ${ }^{a}$ Center for Plasma Astrophysics, Celestijnenlaan 200B, 3001 Leuven, Belgium \\ ${ }^{\mathrm{b}}$ Physics Research Division PINSTECH, P.O. Nilore, Islamabad, Pakistan ${ }^{1}$ \\ ${ }^{\mathrm{c}}$ Department of Physics, COMSATS Institute of Information Technology (CIIT), Islamabad, Pakistan ${ }^{1}$ \\ Received 7 April 2005; received in revised form 4 August 2005; accepted 14 October 2005 \\ Available online 21 October 2005 \\ Communicated by F. Porcelli
}

\begin{abstract}
It is pointed out that the observation of the electrostatic ion acoustic wave frequency can be a suitable check to determine whether the produced plasma is a pure pair-ion plasma or whether it comprises some concentration of electrons. A theoretical model for the pair-ion plasma dynamics is presented along with a new electrostatic mode which can exist only in such systems. It can become unstable in the presence of shear flow and it can give rise to vortex structures in the nonlinear regime. The possibility of shocks and solitons, due to nonlinear drift waves in a pair-ion plasma comprising electrons, is also discussed. The relevance of this investigation to both laboratory and astrophysical plasmas is pointed out.
\end{abstract}

(c) 2005 Elsevier B.V. All rights reserved.

Recently, it has been reported that a pure pair-ion fullerene plasma has been produced in the laboratory [1,2]. Three kinds of electrostatic waves, propagating only in the direction parallel to the external magnetic field have also been discussed [1]. These waves are the ion plasma wave (IPW), the ion acoustic wave (IAW), and the third one has been named as the 'intermediate frequency wave' (IFW) because its frequency lies in between the frequencies of the other two waves, i.e., the IPW and the IAW.

Physically, a pair-ion plasma is similar to an electronpositron plasma. A lot of research work has already been done on electron-positron plasmas which are thought to be generated in astrophysical environments such as in pulsar's magnetospheres [3-6]. Electron-positron plasmas have also been produced experimentally $[7,8]$. The pair-ion plasmas, on the other hand, are an emerging new area of increasing scientific interest.

In this Letter we demonstrate that the observed frequency of the ion acoustic wave can be a suitable check to determine if the plasma is either a pure pair-ion plasma or it contains a sig-

\footnotetext{
* Corresponding author.

E-mail address: saleemhpk@ hotmail.com (H. Saleem).

1 Permanent addresses.
}

nificant concentration of electrons as well. It is further pointed out that the pair-ion plasma dynamics can be somewhat different from ordinary electron-ion plasmas. The modified low frequency modes in magnetized pair-ion plasmas are analyzed and a new low frequency mode which can exist only in such systems is studied both linearly and nonlinearly. A few comments on the Alfvén wave and the instability of the new electrostatic mode in the presence of perpendicular shear flow will be presented. The possibility of nonlinear electrostatic structures will also be pointed out.

Let the magnetic field be constant along the $z$-axis and consider the plasma to be homogeneous. The equation of motion of $\alpha$-specie can be written as

$m_{\alpha} n_{\alpha} \partial_{t} \mathbf{v}_{\alpha}=n_{\alpha} q_{\alpha}\left(\mathbf{E}+\mathbf{v}_{\alpha} \times B_{0} \mathbf{z}\right)-\nabla p_{\alpha}$

where subscript $\alpha= \pm$ denotes positive and negative ions. We assume $\mathbf{E}=-\nabla \varphi$ and $p_{\alpha}=n_{\alpha} T_{\alpha}$. The above equation yields

$$
\begin{aligned}
\left(\partial_{t}^{2}+\Omega_{\alpha}^{2}\right) \mathbf{v}_{\alpha \perp}= & \frac{q_{\alpha}}{m_{\alpha}}\left(\partial_{t} \mathbf{E}+\Omega_{\alpha} \mathbf{E}_{\perp} \times \mathbf{z}\right) \\
& -\frac{\Omega_{\alpha}}{m_{\alpha}} \frac{\nabla_{\perp} p_{\alpha} \times \mathbf{z}}{n_{\alpha}}-\partial_{t} \frac{\nabla_{\perp} p_{\alpha}}{m_{\alpha} n_{\alpha}}
\end{aligned}
$$


and

$\partial_{t} v_{\alpha z}=\frac{q_{\alpha}}{m_{\alpha}} E_{z}-\frac{\partial_{z} p_{\alpha}}{m_{\alpha} n_{\alpha}}$,

where $\Omega_{\alpha}=\frac{e B_{0}}{m_{\alpha}}$. The continuity equation can be written as

$\partial_{t} n_{\alpha}+n_{0 \alpha} \nabla_{\perp} \cdot \mathbf{v}_{\alpha \perp}+n_{0 \alpha} \partial_{z} v_{\alpha z}=0$.

Eqs. (2)-(4) give

$$
\begin{aligned}
& \left\{\omega^{2}\left(\omega^{2}-\Omega_{\alpha}^{2}\right)-v_{T \alpha}^{2} k^{2} \omega^{2}+v_{T \alpha}^{2} k_{2}^{2} \Omega_{\alpha}^{2}\right\} n_{\alpha} \\
& \quad-\frac{n_{0 \alpha} q_{\alpha}}{m_{\alpha}} k_{\perp}^{2} \omega^{2} \varphi-\frac{n_{0 \alpha} q_{\alpha}}{m_{\alpha}}\left(\omega^{2}-\Omega_{\alpha}^{2}\right) k_{z}^{2} \varphi=0 .
\end{aligned}
$$

Writing Eq. (5) for $\alpha= \pm$ and then subtracting one equation from the other, we obtain

$$
\begin{aligned}
& {\left[\omega^{2}\left(\omega^{2}-\Omega_{i}^{2}\right)-v_{T i}^{2} k^{2} \omega^{2}+v_{T i}^{2} k_{z}^{2} \Omega_{i}^{2}\right]\left(n_{+}-n_{-}\right)} \\
& \quad-\left(n_{+}^{0}+n_{-}^{0}\right) \frac{q}{m_{i}} k_{\perp}^{2} \omega^{2} \varphi \\
& \quad-\left(n_{+}^{0}+n_{-}^{0}\right) \frac{q}{m_{i}} k_{z}^{2}\left(\omega^{2}-\Omega_{i}^{2}\right) \varphi=0,
\end{aligned}
$$

where $\Omega_{i}=q B_{0} / m_{i}$ is the ion gyro-frequency. Here, the superscript 0 indicates the equilibrium densities. The magnitude of the charge on both ions is $q$ and they have equal mass $m_{i}$. The temperatures of both ions have also been assumed to be equal, i.e., $T_{+}=T_{-}=T_{i}$ and, hence, we define the ion thermal velocity as $v_{T i}=\left(\gamma_{i} T_{i} / m_{i}\right)^{1 / 2}$. Here, $\gamma_{i}$ is the ratio of specific heats for the adiabatic ions. The Poisson equation reads

$n_{+}-n_{-}=-\frac{\epsilon_{0}}{q} \nabla^{2} \varphi$

We are interested to find a criterium to determine the percentage concentration of electrons in the system. For this we have to investigate the ion acoustic wave frequency and, therefore, we shall also need an electron Boltzmann density distribution which is given as

$n_{e}=n_{0 e} \exp \left(\frac{e \varphi}{T_{e}}\right)$.

The set of equations (6)-(8) yields a few simple but interesting results. Let us discuss the limiting cases one by one.

We observe that a new mode which may be called a finite frequency pair plasma convective cell (PPCC) can exist in such systems in the quasi-neutral approximation. Let us assume that the concentration of electrons is negligible in the system and the net electron momentum exerted on the collective plasma motion can be ignored. In this situation we obtain from Eq. (6), using $n_{+} \simeq n_{-}$, a linear dispersion relation as

$\omega^{2}=\frac{k_{z}^{2}}{k^{2}} \Omega_{i}^{2}$.

It may be noted that this mode requires the condition $k_{z} \ll k$ and, hence, $\omega \ll \Omega_{i}$. Otherwise the ion cyclotron and also the ion plasma waves should appear which do not allow us to assume quasi-neutrality in the absence of electrons.

This mode may exist, in principle, in electron-positron plasmas as well. However, in pulsar magnetospheres since $\omega_{p e} \ll \Omega_{e}$ (where $\omega_{p e}$ and $\Omega_{e}$ are the electron plasma oscillation and gyro-frequencies, respectively) this mode has not been studied yet (to the best of the authors' knowledge). The oscillations of electrons and positrons in the parallel electric field will produce the plasma wave which has a much smaller frequency than the gyro-frequency of these light particles in the strong magnetic field of the order of $10^{12}$ Gauss. Hence, the plasma wave may appear where the quasi-neutrality approximation does not remain valid. In laboratory produced electronpositron plasmas, however, the condition that the electron gyrofrequency is much smaller than electron plasma oscillation frequency, i.e., $\Omega_{e} \ll \omega_{p e}$, generally holds. Nevertheless, a mode with the dispersion relation $\omega=\left[n_{0 e} m_{i} /\left(n_{0 i} m_{e}\right)\right]^{1 / 2}\left(k_{z} / k_{\perp}\right) \Omega_{i}$ has been obtained in the dusty plasma environment. A detailed discussion about this mode and the conditions for its existence can be found in Ref. [9].

When $k_{z}$ and $T_{i}$ are assumed to be zero, and the Poisson equation is used instead of quasi-neutrality in Eq. (6), one obtains $\omega^{2}=\Omega_{i}^{2}+2 \omega_{p i}^{2}$, which is the analogue of the upper hybrid oscillations in electron-ion plasmas. Here, $\omega_{p i}=$ $\left[n_{0} q^{2} /\left(\epsilon_{0} m_{i}\right)\right]^{1 / 2}$ is the ion plasma oscillation frequency, and $n_{0}$ is the background number density of positive and negative ions which are equal in this case.

Now we discuss the purely parallel propagating waves. Assuming quasi-neutrality along with $k_{\perp}=0$ and $B_{0}=0$, Eqs. (6) and (8) yield

$\omega^{2}=\frac{q}{e} N_{0} c_{s}^{2} k_{z}^{2}+v_{T i}^{2} k_{z}^{2}$

where $c_{s}=\left(T_{e} / m_{i}\right)^{1 / 2}$ is the ion sound speed, $N_{0}=1+\frac{\epsilon}{1-\epsilon}$ and $\epsilon=n_{-}^{0} / n_{+}^{0}$. If we have $1 \ll N_{0}$, the frequency of the wave may exceed the ion cyclotron frequency; that is, we may have $\Omega_{i}<\omega$ even if $c_{s} k_{z}<\Omega_{i}$. Here we have $\epsilon=0$ for the case of ordinary electron-ion plasmas and $\epsilon=1$ for the pair-ion plasmas. It may be noticed that for $\epsilon=1 \mathrm{Eq}$. (10) is trivially satisfied if we multiply it with $(1-\epsilon)$ since $c_{s} \rightarrow 0$ as $\epsilon \rightarrow 1$.

The dispersion relation (10) shows that the frequency of the ion acoustic wave will be greater than the ion thermal speed even if $T_{e}=T_{i}$ and $q=e$ due to the first term on the right-hand side which can have $1 \ll N_{0}$. This seems to be the reason that the observed IAW frequency line is above the ion thermal wave plot in Fig. 2 of Ref. [1]. Thus the observation of the IAW frequency can be a test to determine the percentage concentration of electrons in the system. In our opinion, the authors of Ref. [1] have been successful in creating an almost pair-ion plasma but it may be not a perfectly pair-ion plasma since it seems to have $1<N_{0}$.

On the other hand, if $n_{0 e}=0$ and $B_{0}=0$ and we use the Poisson equation along with $k_{\perp}=0$ in Eq. (6), we obtain the ion plasma wave dispersion relation $\omega^{2}=2 \omega_{p i}^{2}+v_{T i}^{2} k_{z}^{2}$.

Now we show that the ion cyclotron wave dispersion relation will be modified in a plasma with the same ions. Let us assume that the plasma is quasi-neutral in the presence of Boltzmann electrons. For simplicity we ignore the ion temperature effects. Eqs. (6) and (8) then yield

$\omega^{4}-\left(\Omega_{i}^{2}+\frac{q}{e} N_{0} c_{s}^{2} k^{2}\right) \omega^{2}+\frac{q}{e} N_{0} c_{s}^{2} k_{z}^{2} \Omega_{i}^{2}=0$. 
In the electron-ion plasma case $N_{0}=1$ and for the ion cyclotron wave we have $k_{z} \ll k_{\perp}$, therefore Eq. (11) yields the well-known dispersion relation $\omega^{2}=\Omega_{i}^{2}+c_{s}^{2} k^{2}$.

In the present situation, $1 \ll N_{0}$ is possible along with $\omega^{2}<$ $N_{0} c_{s}^{2} k_{z}^{2}$, therefore we retain the last term in Eq. (11). It gives for $q=e$,

$$
\begin{aligned}
\omega^{2}= & \frac{1}{2}\left[\left(\Omega_{i}^{2}+N_{0} c_{s}^{2} k^{2}\right)\right. \\
& \left. \pm\left(\left(\Omega_{i}^{2}+N_{0} c_{s}^{2} k^{2}\right)^{2}-4 N_{0} c_{s}^{2} k_{z}^{2} \Omega_{i}^{2}\right)^{1 / 2}\right] .
\end{aligned}
$$

This is the modified ion cyclotron wave dispersion relation. In the limit $\omega \ll \Omega_{i}$ it reduces to the ion acoustic wave:

$\omega^{2}=\frac{q N_{0} c_{s}^{2} k_{z}^{2} / e}{1+q N_{0} \rho_{s}^{2} k^{2} / e}$.

It may also be noted that the low frequency electromagnetic Alfvén waves are not dispersive in a pair-ion plasma. If we assume $n_{e 0}=0$ and use quasi-neutrality because of the low frequency limit along with $\mathbf{E}=-\nabla \phi-\left(\partial A_{z} / \partial t\right) \mathbf{z}$, then we obtain the following Alfvén wave dispersion relation

$\omega^{2}=v_{A}^{2} k_{z}^{2} / 2$,

where $v_{A}^{2}=B_{0}^{2} /\left(\mu_{0} n_{0 i} m_{i}\right)$.

We now investigate the effects of shear flow on the linear and nonlinear dynamics of the PPCC mode. Let there be an external electric field $\mathbf{E}_{0}(x)=-\nabla \varphi_{0} \neq 0$, and let the plasma flow be along the $y$-direction such that both positive and negative ions move with the same shear velocity, i.e., $v_{+}(x)=v_{-}(x)=v_{0}(x)$ and, hence, the background current is zero. The steady state demands $E_{0}=-B_{0} v_{0}$. For the low frequency PPCC mode we ignore the pressure term. Then under drift approximation $\left|\partial_{t}\right| \ll \Omega_{i}$, Eq. (1) gives

$$
\begin{aligned}
\mathbf{v}_{\alpha \perp} & =\frac{1}{B_{0}}\left(\mathbf{E}_{\perp} \times \mathbf{z}\right)-\frac{1}{\Omega_{d}}\left(\partial_{t}+\mathbf{v}_{\alpha} \cdot \nabla\right) \mathbf{v}_{\alpha} \times \mathbf{z} \\
& =\mathbf{v}_{E}+\mathbf{v}_{\alpha p}
\end{aligned}
$$

and

$\left(\partial_{t}+\mathbf{v}_{\alpha} \cdot \nabla\right) v_{\alpha z}=\frac{q_{\alpha}}{m_{\alpha}} E_{z}$,

where $\mathbf{v}_{E}$ is the electric drift and $\mathbf{v}_{\alpha p}$ is the polarization drift for $\alpha$ ions. Note that

$$
\begin{aligned}
\nabla & \cdot\left(\mathbf{v}_{+p}-\mathbf{v}_{-p}\right) \\
& =-\frac{2}{B_{0} \Omega_{\mathrm{I}}}\left[\partial_{t}+\frac{1}{B_{0}} \hat{z} \times \nabla_{\perp}\left(\varphi+\varphi_{0}\right) \cdot \nabla_{\perp}\right] \nabla_{\perp}^{2}\left(\varphi+\varphi_{0}\right) .
\end{aligned}
$$

Therefore, the parallel Eqs. (16) yield

$\left[\frac{\partial}{\partial t}+\frac{1}{B_{0}} \vec{e}_{z} \times \nabla_{\perp}\left(\varphi+\varphi_{0}\right) \cdot \nabla_{\perp}\right]\left(v_{+z}-v_{-z}\right)=-b \frac{\partial \varphi}{\partial z}$,

and the continuity equation can be written as

$$
\begin{aligned}
& {\left[\frac{\partial}{\partial t}+\frac{1}{B_{0}} \vec{e}_{z} \times \nabla_{\perp}\left(\varphi+\varphi_{0}\right) \cdot \nabla_{\perp}\right] a \nabla_{\perp}^{2}\left(\varphi+\varphi_{0}\right)} \\
& \quad=-\frac{\partial}{\partial z}\left(v_{+z}-v_{-z}\right)
\end{aligned}
$$

where $a=-2 /\left(B_{0} \Omega\right)$ and $b=2 q / m_{i}$.

The factor 2 in constants $a$ and $b$ is the effect of pair-ion plasmas. If $\epsilon=0$, i.e., $n_{-}^{0}=0$, then Eqs. (17) and (18) will not remain valid. Rather we shall need electron dynamics with quasi-neutrality $n_{e} \sim n_{i}$ and $m_{e} \rightarrow 0$ to describe the low frequency convective cell. In the case of pair-ion plasmas the nonlinear dynamics of electrostatic convective cell are governed by Eqs. (17) and (18) in the presence of shear flow which can cause a linear instability of PPCC mode.

Assuming a linear perturbation of the form $\varphi(x) \exp \left[i\left(k_{y} y+\right.\right.$ $\left.\left.k_{z} z-\omega t\right)\right]$ we obtain

$\frac{d^{2} \varphi}{d x^{2}}-k_{y}^{2} \varphi+\frac{k_{y} v_{0}^{\prime \prime}}{\omega-v_{0} k_{y}} \varphi-\frac{k_{z} \Omega^{2}}{\left(\omega-v_{0} k_{y}\right)^{2}} \varphi=0$,

where the superscript double prime indicates the second-order differentiation with respect to $x$. Let $\omega=\omega_{r}+i \gamma$, where $\omega_{r}$ and $\gamma$ denote the real and imaginary parts of the frequency, respectively. Then for $\omega_{0}=\left(\omega_{r}-v_{0} k_{y}\right)$ the instability condition turns out to be

$\frac{k_{y} v_{0}^{\prime \prime}}{\omega_{0}^{2}+\gamma^{2}}+\frac{2 k_{z}^{2} \Omega^{2} \omega_{0}}{\omega_{0}^{4}+4 \gamma^{2} \omega_{0}^{2}}=0$.

Let us consider the nonlinear stage of instability in a frame which is moving with velocity components $U_{y}$ and $U_{z}$ in the $y z$-plane. With $\alpha=U_{y} / U_{z}$ Eq. (17) can be written in this frame as

$$
\begin{aligned}
& {\left[\vec{e}_{z} \times \nabla_{\perp}\left(\Phi-B_{0} U_{y} x\right) \cdot \nabla_{\perp}\right]} \\
& \quad \times\left[\left(v_{+z}-v_{-z}\right)-B_{0} \alpha b x\right]=0,
\end{aligned}
$$

where $\Phi=\varphi+\varphi_{0}$. The possible solution of Eq. (20) can be

$\left(v_{+z}-v_{-z}\right)-B_{0} \alpha b x=F\left(\Phi-B_{0} U_{y} x\right)$,

where $F$ is an arbitrary function of the given argument. We choose a linear form of this function $F\left(\Phi-B_{0} U_{y} x\right)=F_{0}(\Phi-$ $B_{0} U_{y} x$ ), with $F_{0}$ an arbitrary constant. Thus the above equation becomes

$v_{+z}-v_{-z}=F_{0} \Phi+B_{0} g x$,

where $g=\alpha b-F_{0} U_{y}$. Similarly, Eq. (18) can be written as

$\nabla_{\perp}\left(\Phi-B_{0} U_{y} x\right) \times \nabla_{\perp}\left(a \nabla^{2} \Phi-F_{0} B_{0} \alpha x\right)=0$,

which yields

$\nabla_{\perp}^{2} \Phi-G_{0} \Phi+\left(G_{0} B_{0} U_{y}-\frac{F_{0}}{a} B_{0} \alpha\right)=0$,

where $G_{0}$ is a constant. It is well known that Eq. (24) admits dipolar and tripolar vortex solutions.

A pair-ion plasma in the presence of electrons can support electrostatic drift waves as well. If drift waves are excited in such a system, shock waves can be produced similar to the electron ion case [10]. We shall here show that the nonlinear electrostatic drift waves can form both shocks and solitons in such plasmas. Then ions continuity equations yield 


$$
\begin{aligned}
\partial_{t} & \Phi-V_{N 0} \partial_{y} \Phi+v_{e} \Phi \partial_{y} \Phi \\
& =\frac{N_{1}}{\Omega_{i}} \partial_{t}\left(D_{e} \nabla_{\perp}^{2} \Phi\right)+N_{2} D_{e} \nabla_{\perp}^{2} \Phi-N_{1} \frac{v}{\Omega_{i}}\left(D_{e} \nabla_{\perp}^{2} \Phi\right),
\end{aligned}
$$

where

$$
\begin{aligned}
& N_{1}=\frac{n_{+}^{0}-n_{-}^{0}}{n_{e 0}}, \quad N_{2}=\frac{v_{i}^{+} n_{+}^{0}-v_{i}^{-} n_{-}^{0}}{\Omega_{i} n_{e 0}}, \\
& V_{N 0}=\frac{D_{e}}{n_{e 0}}\left(\frac{d n_{0}^{+}}{d x}+\frac{d n_{0}^{-}}{d x}\right), \quad D_{e}=\frac{T_{e}}{e B_{0}}, \\
& v_{e}=v_{D T e}-v_{D n e}, \quad v_{D n e}=D_{e} \kappa_{n e}, \quad v_{D T e}=D_{e} \kappa_{T e}, \\
& \kappa_{n e}=\frac{d n_{e 0}}{n_{e 0} d x}, \quad \kappa_{T e}=\frac{d T_{e 0}}{T_{e 0} d x} .
\end{aligned}
$$

The drift wave is assumed to propagate along the $y$-axis. Here $v$ is the collision frequency between positive and negative ions, $v_{i}^{+}=v+v_{0}^{+}, v_{i}^{-}=v+v_{0}^{-}$and $v_{0}^{ \pm}$are the collision frequencies between positive-positive and negative-negative ions, respectively.

We proceed by introducing a new coordinate system moving with constant velocity $U$ along the $y$-direction as $\eta=y-U t$. Eq. (25) in this coordinate can be expressed as the KdV-Burgers equation

$-f(x) \partial_{\eta} \Phi+\frac{1}{2} \partial_{\eta} \Phi^{2}+g(x) \partial_{\eta}^{3} \Phi-\mu(x) \partial_{\eta}^{2} \Phi=0$,

where

$$
\begin{array}{ll}
f(x)=\frac{U+V_{N 0}}{v_{e}}, & g(x)=\frac{N_{1} \rho_{s}^{2} U}{v_{e}}, \\
\mu_{1}=-\frac{N_{1} v \rho_{s}^{2}}{v_{e}}, & \mu_{2}(x)=\frac{\rho_{s}^{2} N_{2}}{v_{e}}, \quad \mu=\mu_{1}+\mu_{2}, \\
c_{s}=\left(\frac{T_{e}}{m_{i}}\right)^{1 / 2}, & \rho_{s}=\frac{c_{s}}{\Omega_{i}} .
\end{array}
$$

Now we look for the solutions of Eq. (26) in different limits. If dispersion is very weak, this equation reduces to the Burgers equation

$-f(x) \partial_{\eta} \Phi+\frac{1}{2} \partial_{\eta} \Phi^{2}-\mu(x) \partial_{\eta}^{2} \Phi=0$,

which admits a monotonic shock solution

$\Phi=f\left[1-\tanh \left(\frac{f}{2 \mu} \eta\right)\right]$.

If dissipation is ignored, Eq. (26) can be expressed as the $\mathrm{KdV}$ equation

$\partial_{t} \Phi+L \Phi \partial_{\eta} \Phi+K \partial_{\eta}^{3} \Phi=0$

which has the following soliton solution

$\Phi=\Phi_{0} \operatorname{sech}^{2}\left(\frac{\eta}{\sqrt{\delta}}\right)$

where $\delta=4 K / U$ and $\Phi_{0}=3 U / L$ are the width and amplitude of the pulse, respectively. Here, $L=f_{0}^{-1}, K=g / f_{0}$, and $f_{0}=$ $\left(1+v_{\text {Dne }} / U\right) / v_{e}$.

On the other hand, if both dissipation and dispersion are retained, the analytical solution has been found by Karpman [11] only in a limiting case. Let us consider Eq. (26) and assume that the solution has a form as $\Phi=\Phi_{0}+\Phi_{1}$, where $\Phi_{1} \ll \Phi_{0}$ as $\eta \rightarrow-\infty$. Then Eq. (26) in linear form becomes

$$
f \Phi_{1}+g \partial_{\eta}^{2} \Phi_{1}-\mu \partial_{\eta} \Phi_{1}=0
$$

The solution of Eq. (31) can be proportional to $\exp (p \eta)$, where

$p=\frac{1}{2}\left[\frac{\mu}{g} \pm\left(\frac{\mu^{2}}{g^{2}}-\frac{4 f}{g}\right)^{1 / 2}\right]$.

Let $\mu_{c}=(4 f g)^{1 / 2}$ be some critical value of dissipative coefficient. Then in the range where $\mu \ll \mu_{c}$ the approximate solution can be written as $\Phi=Q \exp [\mu \eta /(2 g)] \cos \left[(f / g)^{1 / 2} \eta\right]$, where $Q$ is an arbitrary constant. Hence the solution of Eq. (31) becomes

$\Phi=\Phi_{0}+Q \exp \left(\frac{\mu}{2 g} \eta\right) \cos \left(\sqrt{\frac{f}{g}} \eta\right)$.

This solution shows that the form of the nonlinear structure will be like a solitary pulse where dissipation is small. Then, as the dissipation increases, it will take the form of the monotonic shock.

To summarize, we have presented theoretical calculations based on the fluid theory for mainly electrostatic linear and nonlinear waves in magnetized pair-ion plasmas and near pairion plasmas. A new mode seems to appear in pair-ion plasmas which can become unstable in the presence of shear flow. At the nonlinear stage it can form stable vortex structures. This mode has a very low frequency and a large $k_{\perp}$, therefore it can cause cross-field transport similar to other convective cell modes in electron-ion and dusty plasmas. This theoretical model also shows that the observed ion acoustic frequency can be a test to measure $N_{0}$ and hence the percentage concentration of electrons in the system. It is interesting to note that the ion acoustic frequency in a plasma consisting of positive and negative ions with $1<N_{0}$ will be larger than $c_{s} k$. Therefore, even if the electrons are in thermal equilibrium with ions, the ion acoustic wave frequency will be larger than the ion thermal speed which is apparent in Fig. 2 of Ref. [1]. It has also been shown that the nonlinear electrostatic drift waves can form shocks as well as solitons in a near pair-ion plasma depending upon the magnitude of the dispersion and dissipative terms. Electron-positron plasmas are also pair plasma systems for which the dispersion relation (9) becomes $\omega^{2}=k_{z}^{2} \Omega_{e}^{2} / k^{2}$. Electron-positron plasmas have been produced in laboratories and are believed to exist in astrophysical environments as well. Therefore, a further study of this mode can be interesting. On the other hand, experiments are being performed to produce pure pair-ion plasmas. Hence, the present study is relevant to both astrophysical and laboratory plasmas.

\section{References}

[1] W. Oohara, R. Hatakeyama, Phys. Scr. T 116 (2005) 101.

[2] W. Oohara, R. Hatakeyama, Phys. Rev. Lett. 91 (2003) 205005.

[3] N. Iwamoto, Phys. Rev. E 47 (1993) 604.

[4] G.A. Stewart, J. Plasma Phys. 50 (1993) 521.

[5] G.P. Zank, R.G. Greaves, Phys. Rev. E 51 (1995) 6079. 
[6] D.H.E. Dubin, Phys. Rev. Lett. 92 (2004) 195002.

[7] C.M. Surko, T.J. Murphy, Phys. Fluids B 2 (1990) 1372.

[8] R.G. Greaves, M.D. Tinkle, C.M. Surko, Phys. Plasmas 1 (1994) 1439.

[9] P.K. Shukla, A.A. Mamun, Introduction to Dusty Plasma Physics, vol. 1, Institute of Physics, Bristol, 2002.
[10] T. Tasso, Phys. Lett. A 24 (1967) 618.

[11] V.I. Karpman, Nonlinear Waves in Dispersive Media, Pergamon, Oxford, 1975. 